\title{
THE EFFECT OF MOUTHWASH COMBINATION OF IMMUNOGLOBULIN-Y ANTI-COMD STREPTOCOCCUS MUTANS AND CHITOSAN ON THE FORMATION OF STREPTOCOCCUS MUTANS BIOFILM
}

\author{
ENDANG WINIATI BACHTIAR ${ }^{1,2}$, DETA APRITANTIA ${ }^{2}$, AGOENG TJAHAJANI SARWONO2 ${ }^{2}$, BOY M BACHTIAR ${ }^{1,2}$, \\ RETNO D SOEJOEDONO ${ }^{3}$, I WAYAN TEGUH WIBAWAN ${ }^{3}$,
}

${ }^{1}$ Department of Oral Biology, Faculty of Dentistry, Universitas Indonesia, Jakarta, Indonesia. ${ }^{2}$ Oral Science Research Center, Faculty of Dentistry Universitas Indonesia, Jakarta, Indonesia. ${ }^{3}$ Faculty of Veterinary Institute Pertanian Bogor, Indonesia. Email: endang04@ui.ac.id

Received: 10 September 2018, Revised and Accepted: 16 February 2019

\section{ABSTRACT}

Objective: The objective of this study was to investigate the effectiveness of immunoglobulin Y (IgY) antibodies as a dental caries vaccine by utilizing IgY specific to the quorum-sensing signaling receptor ComD of Streptococcus mutans combined with chitosan in the form of mouthwash.

Methods: The effects of a mouthwash containing IgY anti-ComD S. mutans with and without chitosan on biofilm-forming isolates of $S$. mutans were investigated. Subjects were assigned to rinsing twice daily for 6 days with 15-20 ml mouthwash solution for $30 \mathrm{~s}$. Biofilm formation of $S$ mutans isolated from the patiens was measured using a crystal violet method to determine the optical density at $490 \mathrm{~nm}$.

Results: The results indicated that mouthwash containing $\operatorname{IgY}$ anti-ComD S. mutans and chitosan significantly enhanced the biofilm formation of S. mutans. In mouthwash containing IgY anti-ComD S. mutans without chitosan, a reduction in biofilm formation was observed; however, this was not statistically significant.

Conclusions: The mouthwash combination of IgY anti-ComD S. mutans and chitosan enhanced the biofilm formation ability of S. mutans isolated from caries and caries-free subjects. Further, research is needed to determine the appropriate concentrations of IgY anti-ComD S. mutans and chitosan required to effectively inhibit dental caries.

Keywords: Biofilm, Chitosan, IgY, Streptococcus mutans, Mouthwash.

(c) 2019 The Authors. Published by Innovare Academic Sciences Pvt Ltd. This is an open access article under the CC BY license (http://creativecommons. org/licenses/by/4. 0/) DOI: http://dx.doi.org/10.22159/ijap.2019.v11s1.AR170

\section{INTRODUCTION}

A molecular biology-based treatment of Streptococcusmutans, the principal causative agent of dental caries, remains a challenge. Efforts to prevent dental caries using passive immunization methods are ongoing. Initially, attempts for dental caries prevention included active immunization through the administration of whole cell $S$. mutans. However, this was expensive and was considered less effective due to cross-reaction side effects with cardiac muscle tissue [1]. Various investigations are currently underway to develop an alternative method of caries prevention utilizing passive immunization through a topical antibody delivery system.

Immunoglobulin Y (IgY), an antibody found in chicken eggs, has been reported to inhibit the adhesion of $S$. mutans to tooth surfaces [2]. IgY against specific bacteria can be obtained by sensitizing a host chicken with the bacteria. The resultant specific antibodies in the host serum are transferred into the egg yolk as part of maternal immunity. The Faculty of Veterinary Medicine, Bogor Agricultural University, Indonesia, in collaboration with the Faculty of Dentistry, Universitas Indonesia, Indonesia, has produced IgY anti-S. mutans in Single Comb Brown Leghorn laying chickens at a production age of 34 weeks [3]. IgY fraction was purified by processing the chicken egg yolk to produce an IgY anti-S. mutans water-soluble fraction. IgY anti-S. mutans reportedly inhibits the activity of the enzyme that plays an important role in the accumulation and the cariogenicity of S. mutans on tooth surfaces [4].

The role of $S$. mutans in the pathogenesis of dental caries occurs through protein interaction with specific, selective receptors for tooth decay, including glucan-binding protein, glucosyltransferase, and S. mutans I/II antigen [5]. A study investigated the efficacy of IgY as a biofilm inhibitor by routinely applying IgY gel containing whole cell $S$. mutans daily for 56 consecutive days to Sprague-Dawley rats [6]. However, the results were not significant and warranted further research. It was concluded that the efficacy of IgY as a bacterial colonization inhibitor could be improved by utilizing recombinant DNA technology approaches to engineer a vaccine coding for the target protein region of IgY [7]. This has been achieved using recombinant DNA technology to create specific IgY antibodies against signaling peptides (ComD), intermediates, and inter-receptor quorum signals, which are encoded by ComD of $S$. mutans. It has been shown that IgY anti-ComD S. mutans could inhibit inter- and intra-species communication between dental plaque-forming bacteria within certain related components of the quorum-sensing structure. A vaccine containing the ComD coding region of $S$. mutans has been successfully created by Endang Winiati Bachtiar (patent announcement number 0492779.7). Immunization of chickens with this vaccine produces IgY that disrupts the interaction between dental plaque-forming bacteria in S. mutans colonization [8].

Mouthwash can be used as a form of dosage that can utilize IgY anti-ComD S. mutans to prevent dental caries. Mouthwash use aims to reduce oral bacteria and confers a refreshing effect. One of the binders that can be used in mouthwash manufacture is chitosan, a chitin derivative that acts as a natural IgY preservative. In addition, chitosan may play a role in increasing the $\mathrm{pH}$ of the mouth, thereby preventing tooth demineralization. Chitosan has also been reported as antibacterial and an inhibitor of plaque formation in teeth by binding to plaque-forming bacteria [9].

Research concerning the effectiveness and efficiency of IgY anti-ComD S. mutans as a dental caries prevention agent is ongoing, both in vitro 
and in vivo. Research on the combination of IgY anti-ComD S. mutans and chitosan is also in progress to enhance the effectiveness of IgY antiComD S. mutans in various forms of delivery. Therefore, the present study was conducted to investigate the effect of a mouthwash containing combined IgY anti-ComD S. mutans and chitosan on the formation of S. mutans biofilms in caries and caries-free subjects.

\section{METHODS}

\section{Research subjects and preparation}

Subjects were examined for caries risk factors, and plaque samples were taken before the administration of the mouthwash treatment. Subjects were asked to use $15-20 \mathrm{ml}$ mouthwash twice daily for $30 \mathrm{~s}$ for each gargle for 6 consecutive days. Subjects were divided into four groups. In Groups 1 and 2, IgY anti-ComD S. mutans mouthwash was administered in caries and caries-free subjects, respectively. In Groups 3 and 4, combination of IgY anti-ComD S. mutans and chitosan mouthwash was administered in caries and caries-free subjects, respectively.

\section{Mouthwash preparation}

Two kinds of mouthwash were prepared: IgY anti-ComD S. mutans with chitosan and IgY anti-ComD S. mutans without chitosan. The concentration of both IgY anti-ComD S. mutans and chitosan was $0.01 \%$.

\section{Sampling of $S$. mutans specimens}

A cotton bud was applied to the buccal surface of the posterior teeth of the subjects and then immersed into a microcentrifuge tube containing $0.2 \mathrm{ml}$ of sterile phosphate-buffered saline (PBS) solution and pressed against the microcentrifuge tube wall.

\section{Bacterial culture of $S$. mutans}

Samples were cultured on trypticase soy-sucrose-bacitracin (TYS20B) selective solid medium by touching the cotton bud that had been immersed in PBS onto the plate. Each plate was placed inside an anaerobic jar that was filled with an anaerobic gas consisting of balanced $\mathrm{N}_{2}, 10 \% \mathrm{CO}_{2}$, and $10 \% \mathrm{H}_{2}$ for 2 min and subsequently sealed and incubated at $37^{\circ} \mathrm{C}$ for $72 \mathrm{~h}$. Next, sterile toothpicks were used to select 5-6 colonies for culture inoculation into a reaction tube containing $5 \mathrm{ml}$ of selective TYS broth. Each test tube was placed into an anaerobic jar that was filled with anaerobic gas (balanced $\mathrm{N}_{2}, 10 \%$ $\mathrm{CO}_{2}$, and $10 \% \mathrm{H}_{2}$ ) and incubated at $37^{\circ} \mathrm{C}$ for $24 \mathrm{~h}$.

\section{Sample preparation for $S$. mutans biofilm test}

The resultant bacterial cultures of $S$. mutans from the TYS broth were prepared to a concentration of $10^{6} \mathrm{CFU} / \mathrm{ml}$ for each sample. Next, 200 $\mu \mathrm{l}$ of each sample was placed into a well of a 96-well polystyrene plate. This procedure was repeated to obtain two duplicate plates. The plates were placed in an incubator that was filled with anaerobic gas (balanced $\mathrm{N}_{2}, 10 \% \mathrm{CO}_{2}$, and $10 \% \mathrm{H}_{2}$ ) for $2 \mathrm{~min}$ and incubated at $37^{\circ} \mathrm{C}$ for $24 \mathrm{~h}$.

Biofilm formation was measured by determining optical density (OD) at $490 \mathrm{~nm}$

After removal from the incubator, the plate wells were washed with $200 \mu \mathrm{l}$ PBS to remove those bacteria that did not form biofilms at the bottom of the wells. Next, the plate was heated. Following staining with $200 \mu \mathrm{l}$ of $0.1 \%$ crystal violet solution per well, each plate was incubated at $37^{\circ} \mathrm{C}$ for $15 \mathrm{~min}$. Next, the liquid crystal violet was removed, and each well was diluted with $200 \mu \mathrm{l}$ of ethanol. Enzyme-linked immunosorbent assay reader was used to determine the OD at $490 \mathrm{~nm}$ (OD $490 \mathrm{~nm}$ ). Each plate was read twice.

\section{Statistical analysis}

A paired t-test was used to compare the OD $(490 \mathrm{~nm})$ results before and after mouthwash treatment. $p<0.05$ was considered statistically significant.

\section{RESULTS}

There were four subject groups in this study. Two comprised caries subjects and two comprised caries-free subjects who were treated with either IgY anti-ComD S. mutans mouthwash or combination of IgY anti-
ComD S. mutans and chitosan mouthwash, respectively. Each group was tested for the presence of $S$. mutans biofilms before and after the mouthwash treatment. Following sample preparation and treatment as described above, the differences in the OD $(490 \mathrm{~nm})$ were observed, which were a direct reflection of the degree of biofilm formation. The higher the OD $(490 \mathrm{~nm})$, the greater the biofilm formation.

In the caries and caries-free groups treated with the mouthwash containing a combination of IgY anti-ComD S. mutans and chitosan, the biofilm OD $(490 \mathrm{~nm})$ exhibited a significant increase after treatment $(\mathrm{p}=0.035$ and $\mathrm{p}=0.046$, respectively) (Figs. 1 and 2). In the caries subjects treated with mouthwash containing IgY anti-ComD S. mutans, OD $(490 \mathrm{~nm})$ decreased following treatment (Fig. 3). However, this result was not statistically significant. A similar trend was observed in non-caries subjects treated with a mouthwash containing IgY antiComD S. mutans, but the results were also not significant (Fig. 4).

\section{DISCUSSION}

Biofilms comprise communities of various bacterial species that communicate with each other. Biofilm disruption could potentially be achieved by the enhancement of IgY as a bacterial colonization inhibitor utilizing a recombinant DNA technology approach to engineer a vaccine coding for the target protein region of IgY [8]. DNA recombinant could be created by producing specific IgYs for peptide signals as well as intra- and inter-species quorum signal receptors, which are encoded by ComD in S. mutans. IgY anti-ComD S. mutans should block the intra- and inter-species communication of dental plaque-forming bacteria with specific interventions against the ComD molecules that play a role in quorum-sensing mechanisms. The immunization of chickens with

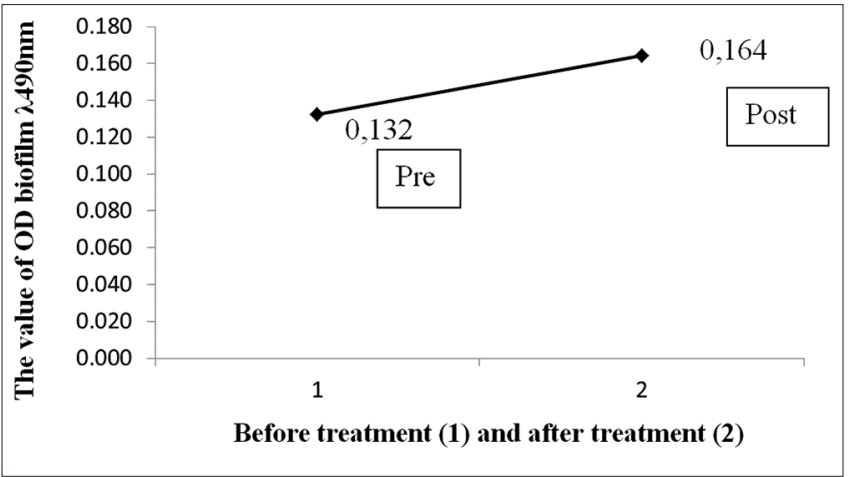

Fig. 1: Biofilm mass/OD (490 $\mathrm{nm})$ in caries subjects before and after treatment with mouth rinse combination of IgY antiComD Streptococcus mutans and chitosan. $p=0.035 ; p<0.05$ was considered statistically significant

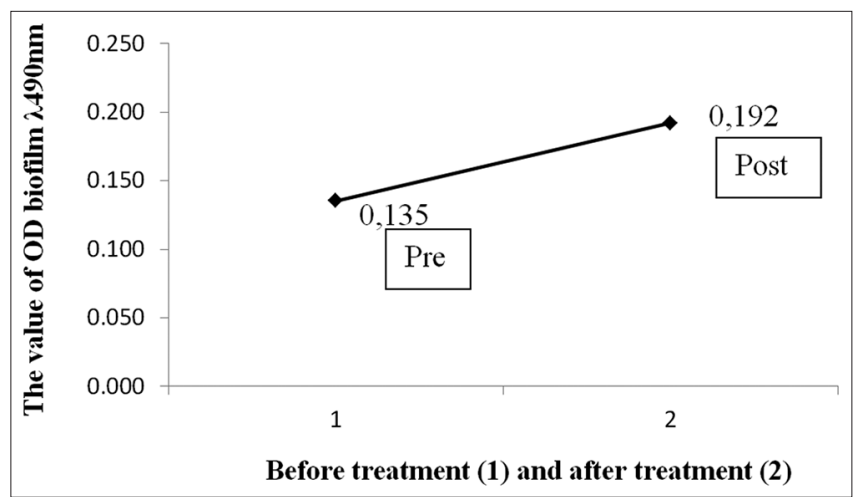

Fig. 2: Biofilm mass/OD (490 nm) in caries-free subjects before and after treatment with mouth rinse containing a combination of IgY anti-ComD Streptococcus mutans and chitosan. $\mathrm{p}=\mathbf{0 . 0 4 6}$; $\mathbf{p}<\mathbf{0 . 0 5}$ was considered statistically significant 




Fig. 3: Biofilm mass/OD (490 $\mathrm{nm})$ in caries subjects before and after treatment with mouthwash containing IgY antiComD Streptococcus mutans. $\mathrm{p}=0.375 ; \mathrm{p}<0.05$ was considered statistically significant

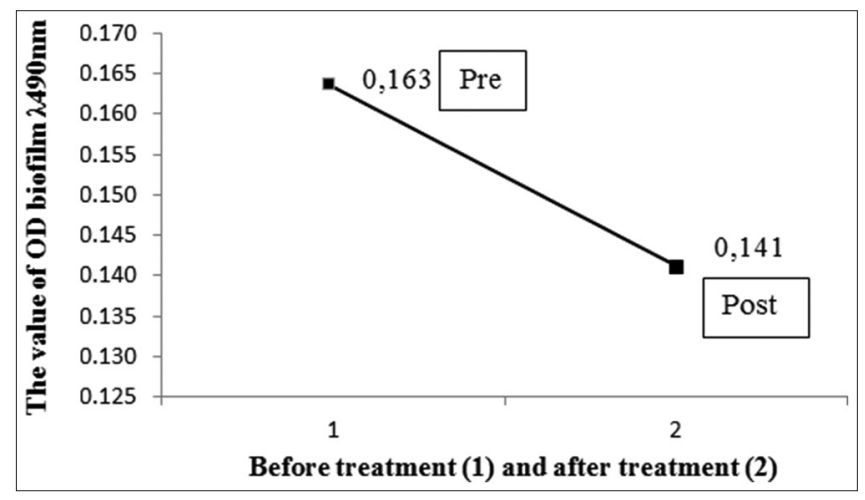

Fig. 4: Biofilm mass/OD $(490 \mathrm{~nm})$ in caries-free subjects before and after treatment with mouthwash containing IgY antiComD Streptococcus mutans. $\mathrm{p}=0.408 ; \mathrm{p}<0.05$ was considered statistically significant

a vaccine containing the ComD coding region of $S$. mutans stimulates the production of IgY that could interfere with the interaction between dental plaque bacteria, particularly during S. mutans colonization [7].

This study determined the effect of a mouthwash containing a combination of IgY anti-ComD S. mutans and chitosan on the biofilm formation ability of $S$. mutans isolated from caries and caries-free subjects. Following treatment, the mouthwash combination of IgY antiComD S. mutans and chitosan significantly increased the biofilm OD 490 $\mathrm{nm}$, indicating an increase in biofilm formation. The opposite occurred in subjects treated with IgY anti-ComD S. mutans without chitosan. The biofilm OD $(490 \mathrm{~nm})$ decreased after treatment, indicating a decrease in biofilm formation. However, the test results were not statistically significant.

The observed increase in biofilm OD in subjects treated with the mouthwash combination of IgY anti-ComD $S$. mutans and chitosan may be related to the properties and characteristics of IgY anti-ComD S. mutans and chitosan. IgY is a protein that is susceptible to acidic conditions and a $\mathrm{pH}<4.0$, whereas chitosan is a soluble polymer in acidic solutions $[1,10]$. Thus, the conditions needed to dissolve chitosan could decrease the effectiveness of IgY anti-ComD S. mutans in biofilm inhibition. The $\mathrm{pH}$ of the mouthwash containing $\operatorname{IgY}$ anti-ComD S. mutans without chitosan is 5.7, and IgY remains stable at $\mathrm{pH}$ 4.0-9.0. Furthermore, increased biofilm formation may occur due to changes in the biological properties of IgY anti-ComD S. mutans caused by chitosan.

Research on the effect of the combination of IgY anti-S. mutans and chitosan on biofilm formation in vitro was conducted in 2009 by Tri [11], who reported decreased serotype biofilm formation, but the results were not statistically significant. A study by Febyani [12] on the effects of IgY anti-S. mutans and chitosan toward the autoaggregation of $S$. mutans serotypes c, d, e, and $\mathrm{f}$ demonstrated an increased autoaggregation of $S$. mutans serotypes $\mathrm{c}$ and $\mathrm{e}$ in the treatment combination of IgY anti-S. mutans and chitosan.

The increased biofilm formation that was observed following treatment with a mouthwash combination of IgY anti-ComD S. mutans and chitosan in this study was influenced by many factors including the differences in characteristics possessed by IgY anti-ComD S. mutans and chitosan. Both are reported to have antibacterial effects that could interfere with the activity of $S$. mutans in biofilm formation. However, based on the results of this study, IgY anti-ComD S. mutans combined with chitosan in mouthwash increases biofilm formation. This could also be caused by unbalanced concentrations of IgY anti-ComD S. mutans and chitosan in the mouthwashes. In addition, caries-risk factors in the subjects may also affect the formation of biofilms. However, examination of these risk factors in the study subjects revealed that all subjects demonstrated a low level of caries-risk factors. Other mouthwash components that have antibacterial functions but do not specifically attack $S$. mutans bacteria may also affect the effectiveness of IgY anti-ComD S. mutans and chitosan in inhibiting $S$. mutans biofilms. This study provides an overview of the effect of mouthwash combination of IgY anti-ComD S. mutans and chitosan on the ability of $S$. mutans isolated from caries and caries-free subjects to form biofilms. However, further, research regarding the appropriate concentrations of IgY anti-ComD S. mutans and chitosan is necessary to effectively inhibit dental caries. Investigations also need to be performed to prove chitosan as an effective binding agent for IgY anti-ComD S. mutans in the dosage form of mouthwash through observation of IgY protein quality through western blot analysis.

\section{CONCLUSIONS}

The results showed that the mouthwash combination of IgY antiComD S. mutans and chitosan enhanced the biofilm formation ability of S. mutans isolated from caries and caries-free subjects.

\section{CONFLICTS OF INTEREST}

The authors report no conflicts of interest.

\section{ACKNOWLEDGEMENT}

This study was supported by the Competence-based Grant from the Directorate Higher Education, Ministry of Education Republic of Indonesia 2013-2014

\section{REFERENCES}

1. Karlsson M, Larsson A. Chicken IgY: Utilizing the evolutionary advantage. Worlds Poult Sci J 2004;60:341-8.

2. Shivakumar KM, Vidya SK, Chandu GN. Dental caries vaccine. Indian J Dent Res 2009;20:99-106.

3. Widjaja A. Yolk imunoglobulin (IgY) to prevent tooth caries. M I Ked Gigi 2008;23:90

4. Nguyen SV, Icatlo FC Jr., Nakano T, Isogai E, Hirose K, Mizugai H, et al. Anti-cell-associated glucosyltransferase immunoglobulin Y suppression of salivary mutans streptococci in healthy young adults. J Am Dent Assoc 2011;142:943-9.

5. Napimoga MH, Höfling JF, Klein MI, Kamiya RU, Gonçalves RB. Transmission, diversity and virulence factors of Streptococcus mutans genotypes. J Oral Sci 2005;47:59-64.

6. Bachtiar EW, Afdhal A, Meidyawati R, Soejoedono RD, Poerwaningsih E. Effect of topical anti Streptococcus mutans igY gel on quantity of $S$. mutans on rats' tooth surface. Acta Microbiol Immunol Hung 2016;63:159-69.

7. Bachtiar EW, Soejoedono RD, Bachtiar BM, Henrietta A, Farhana N, Yuniastuti M, et al. Effects of soybean milk, chitosan, and anti Streptococcus mutans igY in malnourished rats' dental biofilm and the igY persistency in saliva. Interv Med Appl Sci 2015;7:118-23.

8. Bachtiar EW, Bachtiar BM, Soejoedono RD, Wibawan IW, Afdhal A. Biological and immunogenicity property of igY anti $S$. Mutans comD. Open Dent J 2016;10:308-14. 
9. Hennen WJ. Chitosan. Pleasant Grove, Utah: Woodland Publishing Inc.; 1996.

10. Dutta PK, Dutta J, Tripathi VS. Chitin and chitosan: Chemistry, properties and applications. J Sci Ind Res 2004;63:20-31

11. Tri AA. Solution Combination Effects of Imunoglobulin Y Anti Streptococcus mutans and Chitosan Towards Biofilm Formation
Process Streptococcus mutans (Serotype c, e, f) and Streptococcus sobrinus (Serotype d) in-vitro. [Dissertation]. Jakarta: Universitas Indonesia; 2009.

12. Febyani N. Efect of Immunoglobulin Y (IgY) Anti Streptococcus mutans and Chitosan Towards Streptococcus mutans Serotype c, d, e dan f. [Dissertation]. Jakarta: Universitas Indonesia; 2009. 